\title{
Analysis of cranial neural crest migratory pathways in axolotl using cell
}

\section{markers and transplantation}

\author{
Hans-Henning Epperlein'1, Daniel Meulemans², Marianne Bronner-Fraser², Herbert Steinbeisser ${ }^{3}$ and \\ Mark A. J. Selleck4,* \\ ${ }^{1}$ Institut für Anatomie, Techn.Universität, Fetscherstrasse 74, D-01307 Dresden, Germany \\ 2Division of Biology and Beckman Institute, 139-74 California Institute of Technology, Pasadena, CA 91125, USA \\ 3Max-Planck-Institut für Entwicklungsbiologie, Spemannstrasse 35, D-72076 Tübingen, Germany \\ ${ }^{4}$ Department of Cell and Neurobiology, University of Southern California Keck School of Medicine, 1333 San Pablo Street, \\ BMT 401, Los Angeles, CA 90089, USA \\ *Author for correspondence (e-mail: mselleck@hsc.usc.edu)
}

Accepted 3 April; published on WWW 23 May 2000

\section{SUMMARY}

We have examined the ability of normal and heterotopically transplanted neural crest cells to migrate along cranial neural crest pathways in the axolotl using focal DiI injections and in situ hybridization with the neural crest marker, AP-2. DiI labeling demonstrates that cranial neural crest cells migrate as distinct streams along prescribed pathways to populate the maxillary and mandibular processes of the first branchial arch, the hyoid arch and gill arches 1-4, following migratory pathways similar to those observed in other vertebrates. Another neural crest marker, the transcription factor AP-2, is expressed by premigratory neural crest cells within the neural folds and migrating neural crest cells en route to and within the branchial arches. Rotations of the cranial neural folds suggest that premigratory neural crest cells are not committed to a specific branchial arch fate, but can compensate when displaced short distances from their targets by migrating to a new target arch. In contrast, when cells are displaced far from their original location, they appear unable to respond appropriately to their new milieu such that they fail to migrate or appear to migrate randomly. When trunk neural folds are grafted heterotopically into the head, trunk neural crest cells migrate in a highly disorganized fashion and fail to follow normal cranial neural crest pathways. Importantly, we find incorporation of some trunk cells into branchial arch cartilage despite the random nature of their migration. This is the first demonstration that trunk neural crest cells can form cartilage when transplanted to the head. Our results indicate that, although cranial and trunk neural crest cells have inherent differences in ability to recognize migratory pathways, trunk neural crest can differentiate into cranial cartilage when given proper instructive cues.

Key words: DiI, AP-2, Cell movement, Cartilage differentiation, Branchial arches, Axolotl, Neural crest

\section{INTRODUCTION}

The neural crest has long attracted the attention of developmental biologists because these cells migrate over extensive distances to reach a variety of stereotyped target sites and subsequently give rise to a plethora of different cell types, including sensory neurons, autonomic neurons, glial cells, pigment cells, endocrine cells and connective and supportive tissue (Le Douarin, 1982; Hall and Hörstadius, 1988). It is well established that some derivatives arise only from restricted positions along the rostrocaudal axis. For instance, sympathetic neurons are derived solely from trunk and upper lumbar neural crest, whereas parasympathetic neurons are generated from hindbrain and sacral neural crest cells. Additionally, only cranial neural crest progenitors will form supportive tissues of the head, such as cartilage and bone (Le Douarin, 1982).

How does the neural crest generate such diverse derivatives that vary according to their rostrocaudal position of origin?
One possibility is that the premigratory neural crest may be a heterogeneous population, containing single cells that are committed to a specific lineage or to a subset of neural crest derivatives. In this model, neural crest cells might choose their migratory pathways in accordance with specific environmental cues, as is the case during pigment pattern formation of certain amphibian embryos (Epperlein and Löfberg, 1990). According to this model, rostrocaudal differences in neural crest derivatives would be due to the presence or absence of specific neural crest precursors in a region-specific manner.

An alternative possibility, supported by various data, is that neural crest cells are initially multipotent and become progressively committed to their fates as they migrate to, or after they reach, their destinations. Evidence comes from experiments in the trunk of chick embryos where individual neural crest precursors were shown to contribute to multiple neural crest derivatives (Bronner-Fraser and Fraser, 1988; 1989; Selleck and Bronner-Fraser, 1995). Furthermore, clonal 
analyses in tissue culture support the idea that single cranial neural crest cells can form derivatives as diverse as neurons and cartilage (Baroffio et al., 1991). It appears that the fate of such multipotent precursors can be influenced by a variety of growth factors, including BMPs and neuregulins, which induce adrenergic (Varley et al, 1995; Shah et al., 1996; Reissman et al., 1996) and glial derivatives (Shah et al., 1994), respectively.

Possible explanations for the rostrocaudal difference in cartilage-forming ability of the neural crest include (i) regionspecific differences in pathway/target-derived instructive cues, and/or (ii) premigratory differences between cranial and trunk neural crest precursors with respect to their chondrogenic potential. Thus, neural crest cells in the head may differentiate into cartilage because the developing head contains cartilageinducing signals not present at other levels of the developing embryo. Indeed, the importance of pharyngeal endoderm for the differentiation of visceral cartilage from cranial neural crest cells has been shown in urodele embryos in vivo (Hörstadius and Sellman, 1946) and in vitro (Epperlein and Lehmann, 1975; Graveson and Armstrong, 1987). There appears to be no such cartilage-inducing signal in the trunk.

While these results suggest that pathway- or target-specific cues influence neural crest differentiation into cartilage, there is also evidence for differences in chondrogenic potential between cranial and trunk neural crest. In one classic study on urodele embryos (Hörstadius and Sellman, 1946), a preliminary fate map of the cranial crest was made using vital dyes to follow the normal patterns of cranial neural crest cell migration (see Fig. 1). Hörstadius and Sellman's main finding was that neural crest cells destined for different branchial arches (the mandibular arch, the hyoid arch and four gill arches) arise from specific rostrocaudal levels of the neural folds. Using heterotopic grafting of head and trunk neural fold fragments, they challenged the fate of different neural crest populations and found that trunk neural crest grafted into the head fails to migrate in an orderly fashion and was not observed to form cartilage, confirming the results of Raven (1931). Furthermore, pharyngeal endoderm was not able to induce cartilage formation from neural crest cells when grafted to the trunk (Hörstadius and Sellman, 1946). Together, these data suggest that cranial neural crest differentiates into cartilage, but trunk crest will not, because of intrinsic differences in chondrogenic potential between the two, in addition to specific pathway-derived cues present only in the head.

Because of the wealth of experimental embryology performed on the neural crest in urodeles, this system bears revisiting using improved vital dyes and newly available molecular markers. The axolotl embryo offers many advantages over other vertebrate embryos for examining the potential and commitment of neural crest cells, because at early stages of development the entire cranial neural plate and neural folds are distinct and amenable to extensive microsurgical manipulation. In this study, we have constructed a new fate map of the cranial neural crest in the axolotl using two complementary methods for cell labeling. In some experiments, neural fold cells were labeled with DiI, a lipophilic fluorescent dye that has been used extensively (Honig and Hume, 1989) in a number of species to follow neural crest migration and derivatives (Serbedzija et al, 1989; 1992; Collazo et al., 1993). As a second marker, we have examined cranial neural crest cell migration by the expression of AP-2, a transcription factor expressed by this population (Mitchell et al., 1991; Shen et al., 1997).

In addition to confirming most aspects of the fate map of Hörstadius and Sellman (1946), we provide evidence for a segmental origin and migration of neural crest cells from the hindbrain. Grafting experiments within the rhombencephalic (posterior cranial) neural folds demonstrate that a small transposition of neural crest leads to cells migrating to a new branchial arch, suggesting that premigratory neural crest are not committed to a specific target site. In other experiments, we rotated entire cranial neural folds and found that when cells are displaced far from their original location, they appear unable to respond appropriately to their new milieu. Most notably, when trunk neural folds are heterotopically grafted into the head, they migrate in a highly disorganized fashion and fail to follow normal cranial crest pathways. Despite the random nature of their migration, we find incorporation of some trunk cells into branchial arch cartilage. These results suggest that, despite inherent differences in migratory ability between head and trunk neural crest cells, trunk neural crest can differentiate into cranial cartilage when given proper instructive cues.

\section{MATERIALS AND METHODS}

\section{Embryos}

Wild-type (dark, D/-), white mutant (dd), and albino (aa) embryos of the Mexican axolotl (Ambystoma mexicanum) were obtained from the axolotl colony in Bloomington, Indiana, USA. The embryos were kept in tap water at room temperature or at $7-8^{\circ} \mathrm{C}$ and were staged according to the normal table of Bordzilovskaya et al. (1989).

\section{Dil injections into neural folds}

CellTracker CM-DiI (C-7000; Molecular Probes) and CellTracker Green CMFDA (C-7025; Molecular Probes) were dissolved in absolute ethanol to a concentration of $1 \mathrm{mg} / \mathrm{ml}$ and further diluted in 4 or 9 parts of $10 \%$ sucrose in water just before starting the injections. Glass micropipettes were backfilled with the DiI solution and attached to a Parker Hannifin Corporation Picospritzer II assembly. DiI was injected into (A) selected parts of the head neural fold of dark neurulae (stage 15/16) or (B) grafted head or trunk neural fold fragments of dark neurulae (stages 15-17). CellTracker Green was used only in posterior head neural fold reversals (see below).

\section{A. Cranial neural crest fate mapping experiments}

Embryos (stage 15/16) were dipped briefly in $70 \%$ ethanol, washed thoroughly with sterile Steinberg solution (Steinberg, 1957), decapsulated mechanically and placed into the hollow of an agar dish filled with Steinberg saline.

Labeling of the entire cranial neural folds with Dil (6 cases)

Zones 1-7 (Figs 1 and 5) of the left or right head neural fold were labeled with DiI to examine the movement of the entire neural crest population. DiI injections were monitored with a Leica MZFLIII epifluorescence stereomicroscope. 1-2.5 days after injection, living embryos were observed with an epifluorescence microscope. Fluorescent and bright-field images were captured and processed as described below.

\section{Focal injection of Dil into three distinct sites in a single} embryo (6 cases)

DiI was focally injected at three separate sites (zones 3, 4 and 6) of the cranial neural fold in order to investigate migration and 
pathfinding of small groups of neural crest cells in living embryos. Images of fluorescently labeled cells were obtained and processed as described below.

\section{Focal injections of Dil into a single site (12 cases)}

DiI was injected into one or three adjacent spots at zones 4-7 of the head neural fold in order to study internal migration and localization of neural crest cells in transverse sections. 1 (stage 25), 2 (stage 30/31) and 3 days (stage 35/36) after the injection, embryos were fixed in $4 \%$ paraformaldehyde (PFA) in $0.1 \mathrm{M}$ PBS overnight. After washing in PBS, vibratome sections were made, stained as indicated below ('histology') and examined with an epifluorescence microscope.

\section{B. Injections into grafted tissue}

For grafting experiments, neurulae were sterilized as indicated above. Head or trunk neural fold fragments were removed under sterile conditions using tungsten needles and grafted to the appropriate location. After a short time, to permit healing of the operated region, DiI was expelled onto the surface of the graft while viewed under epifluorescence optics.

\section{Heterotopic grafting experiments}

\section{A. Head neural fold reversals}

Three types of head neural fold reversals were carried out at the neurula stage (stage 15) of dark embryos. (a) Anterior head neural fold reversals (5 cases): fragments comprising zones 1-4 were explanted and inserted in the reversed orientation $(4 \rightarrow 1 ; 4$ anterior, 1 posterior); (b) posterior head neural fold reversals (16 cases): fragments comprising zones 5-7 were excised and inserted in the reversed orientation $(7 \rightarrow 5 ; 7$ anterior, 5 posterior); (c) unilateral full length cranial fold reversals ( 8 cases): the right cranial neural fold (zones 1-7) was excised and regrafted in a reversed orientation to the left side of a host embryo and vice versa $(7 \rightarrow 1 ; 7$ anterior, 1 posterior). In all experiments, the graft was subsequently labeled with DiI. In most cases, the entire length of the grafted fold was labeled, while in other experiments, two spots of DiI were made into the transplanted fold. In some of the posterior cranial neural fold reversals, DiI injections were made into the new anterior end of the graft and CellTracker Green was injected at the new posterior end.

\section{B. Trunk neural fold grafts to head (about 8 cases)}

Mid-trunk neural fold fragments from donor neurulae (stages 15-18) were grafted heterotopically into zones 5-7 of host embryos from which these cranial areas had been extirpated. The grafts were injected with DiI shortly after the operation. Embryos were grown to desired stages (up to stage 34 or 38) and the distribution of DiI-labeled cells was examined both in wholemounts and transverse vibratome sections. In some cases, sections were also labeled with a mesodermal marker (12/101) or an anti-6-chondroitin sulfate proteoglycan antibody.

\section{Cloning and sequence analysis of Ambystoma AP-2}

cDNA was synthesized from stage 22-35 Ambystoma embryo total RNA by poly-T-primed reverse transcription and used as a template for PCR. The following degenerate primers were designed against conserved regions of vertebrate and Drosophila AP-2 genes and used to amplify a $522 \mathrm{bp}$ fragment of the Ambystoma AP-2 homolog:

AP-25'1 = 5'GTRTTCTGYKCAGKYCCYGGICG 3' and

AP- $23^{\prime} 1=5^{\prime}$ GWKATVAGGKWGAAGTGSGTCA $3^{\prime}$.

The fragment was TA cloned using the pGEM-T easy kit (Promega), sequenced, and compared to known AP-2 genes using the Megalign program.

\section{In situ hybridization}

Sense and antisense riboprobes were sythesized from the cloned fragment (DIG RNA labeling kit, Roche diagnostics). In situ hybridization was performed on albino axolotl embryos (about 40 embryos at stages 15-37) as described by Henrique et al. (1995) with the addition of an extra wash in MAB-T overnight at $4^{\circ} \mathrm{C}(100 \mathrm{mM}$ maleic acid, $150 \mathrm{mM} \mathrm{NaCl}, \mathrm{pH}$ 7.5, 0.1\% Tween 20). Hybridization was performed at $65^{\circ} \mathrm{C}$.

\section{Histology/immunostaining}

Transverse $100 \mu \mathrm{m}$ sections were cut through DiI-labeled/ formaldehyde-fixed embryos and through in situ hybridized (AP-2) embryos, using a Vibratome Series 1000 sectioning system (Ted Pella, Inc.). Sections found to contain DiI-labeled cells were, in most cases, counterstained with an anti-fibronectin antibody to visualize tissue borders. Sections were incubated first with a polyclonal antifibronectin antibody (Dako, Hamburg), then with an FITC-conjugated goat-anti-rabbit secondary antibody (Dianova, Hamburg). These sections were also stained with DAPI $(0.1-1 \mu \mathrm{g} / \mathrm{ml}$ in PBS) to mark cell nuclei. To determine whether any mesodermal cells were inadvertently included in trunk neural fold fragments grafted to the head, vibratome sections $(100 \mu \mathrm{m})$ were stained with the $12 / 101$ monoclonal antibody (a skeletal muscle marker; DSHB, Iowa, USA), followed by an FITC-conjugated goat anti-mouse antibody. In some instances, DiI-labeled cells were found within cartilaginous condensations and a number of such sections were immunostained with an anti-6-chondroitin sulfate proteoglycan antibody (anti 6CSPG; ICN, Eschwege). Vibratome sections were pre-treated with chondroitinase $\mathrm{ABC}$ ( 2 units $/ \mathrm{ml}$ ) for 45 minutes, followed by a 1 hour incubation in anti 6-CSPG antibody. Sections were then incubated in a FITC-conjugated goat anti-mouse IgG secondary antibody.

\section{Image analysis}

Whole embryos and sections were analyzed with an epifluorescence microscope and images were recorded with a Spot camera. Using Adobe Photoshop software, the contrast and brightness of captured images were optimized, and bright-field images of whole embryos were combined with the corresponding fluorescence (DiI) images. Separate images were captured from sectioned material for DiI labeling (neural crest cells), anti-fibronectin staining (tissue borders), anti-6-CSPG staining (cartilaginous condensations), 12/101 staining (trunk mesoderm) and DAPI staining (cell nuclei), before being superimposed to produce a single image.

\section{RESULTS}

\section{Migratory pathways of cranial neural crest in the Mexican axolotl}

We performed a series of experiments to confirm the accuracy of the Hörstadius and Sellman (1946) cranial neural crest fate maps (Fig. 1), using modern dye-labeling techniques. Cranial neural fold cells were marked with DiI, either by labeling the entire neural folds, or by making small focal injections of DiI into small groups of cells.

\section{Whole neural fold labeling with Dil}

In six different dark neurulae (stage 15/16), the entire left head neural fold (corresponding to zones 1-7 in Fig. 1) was labeled with a contiguous line of DiI (Fig. 2A). Approximately 1, 1.5, 2 and 2.5 days after dye injection, the distribution of DiI in the developing embryos was examined and recorded.

At various time points after labeling it was possible to distinguish (i) a relatively wide, longitudinal band of labeled cells on the dorsum of the embryo and (ii) a number of distinct narrow streams of labeled cells emanating from the dorsal neural tube. The dorsal band of labeled cells comprised of derivatives of the labeled neural fold, including epidermal 
Fig. 1. Fate map of the neural crest cells in axolotl (modified from Hörstadius and Sellman, 1946). (A) A drawing of a stage 16 embryo describing the sites of vital dye labeling. Cranial and anterior trunk neural folds were marked unilaterally with alternating spots of vital dyes, Nile Blue sulfate and Neutral Red. (B) Stage 33; the prospective fate of neural crest cells is indicated and divided into arbitrary zones. Zones 4-7 give rise to the six

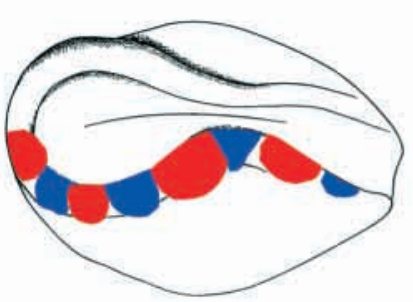

A

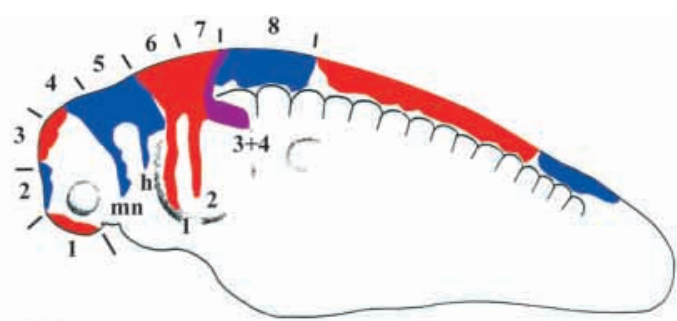

B

branchial arches of the visceral skeleton: mandibular arch (mn), hyoid arch, (hy), and four gill arches (1-4). Zones 1, 2 and 8 are nonskeletogenic, while 3 gives rise to anterior trabeculae (Hall, 1999).

cells, neural tube cells and premigratory neural crest precursors. As development proceeded, the rostrocaudal extent of labeled cells in this band appeared to increase, presumably as a consequence of convergence and extension movements.

Neural crest cells appeared as four dorsoventrally oriented streams of cells. Each was distinct and separated from the others, at least along the distal two-thirds of their migratory pathway, on the ventral aspect of the embryo. The streams appeared in a temporal sequence, with anterior-most neural crest cells migrating prior to posterior-most cells.

About 1 day after dye injection, neural crest cells were observed migrating around the anterior and posterior margins of the eye (Fig. 2B). Whereas the anterior-most cells probably contribute to tissues of the eye itself and adjacent anterior head mesenchyme, the posterior stream contains putative first branchial arch (maxillary and mandibular) neural crest cells. 1.5 days after dye injection, first branchial arch neural crest remained as a coherent stream en route to a peri-stomodeal location. A second, putative hyoid neural crest stream could be seen posterior to the first branchial arch stream (Fig. 2C), followed by a third ( $1^{\text {st }}$ gill arch) stream. 2 days after dye injection (Fig. 2D), the first branchial arch stream appeared to diverge into two tongues in the vicinity of the developing mouth (presumably into separate maxillary and mandibular arch neural crest cells). In addition to the hyoid stream (which now extends to the ventral portion of the embryo), 2-3 streams of neural crest could be seen advancing into the gill bulges. After 2.5 days (stage 35), the maxillary and mandibular stream became still more distinct, but the general pattern remained unchanged (data not shown).

Although our whole neural fold labeling experiments provided evidence that neural crest cells do not mix along the

Fig. 2. Continuous and focal labeling of the head neural fold of axolotl neurulae (stage 15/16) with DiI. (A) For labeling the entire neural folds, DiI was injected throughout zones 1-7 (see Fig. 1) on the left side. B-D illustrate the distribution of labeled cells approximately 1 day, stage 24 (B), 1.5 days, stage 28 (C), and 2 days, stage 31 (D) after dye injection. (E) For focal labeling, zones 3, 4 and 6 were injected with small amounts of DiI. F-H illustrate the distribution after focal labeling approximately 1 day, stage $22(\mathrm{~F})$, 1.5 days, stage $30(\mathrm{G})$ and 2.5 days, stage $35(\mathrm{H})$ after dye injection. The small arrowhead in $\mathrm{B}$ indicates the optic vesicle and the large arrowhead is the gill bulge. In C, G, and $\mathrm{H}$, the optic vesicle is indicated by a small arrowhead. In B-D different embryos are shown, whereas in F-H a single embryo is recorded at the different time points. mx, maxillary arch; mn, mandibular arch; h, hyoid arch; 1, 2, 3: gill arches $1,2,3$. distal parts of their migratory pathway, we were unable to determine whether mixing of neural crest cells occurs immediately after emigration from the neural folds, in the proximal part of the pathway. One reason for this is that labeled neural folds give rise to epidermal cells, which migrate over the dorsal part of the embryo, making it difficult to visualize underlying labeled neural crest cells.

\section{Focal injection of Dil into the cranial neural folds}

While DiI labeling of the entire cranial neural fold provides a good over-view of neural crest migration in the head, it gives
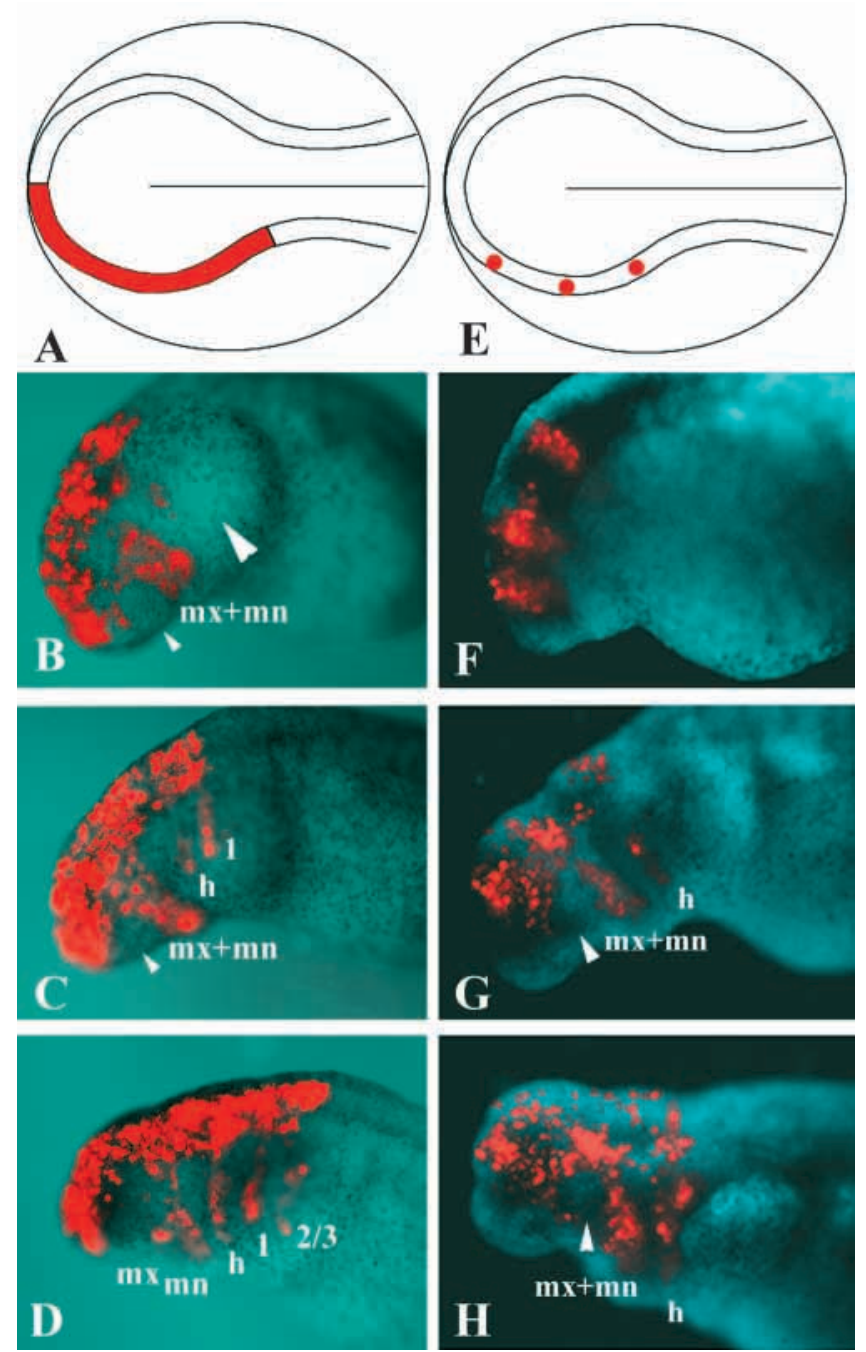

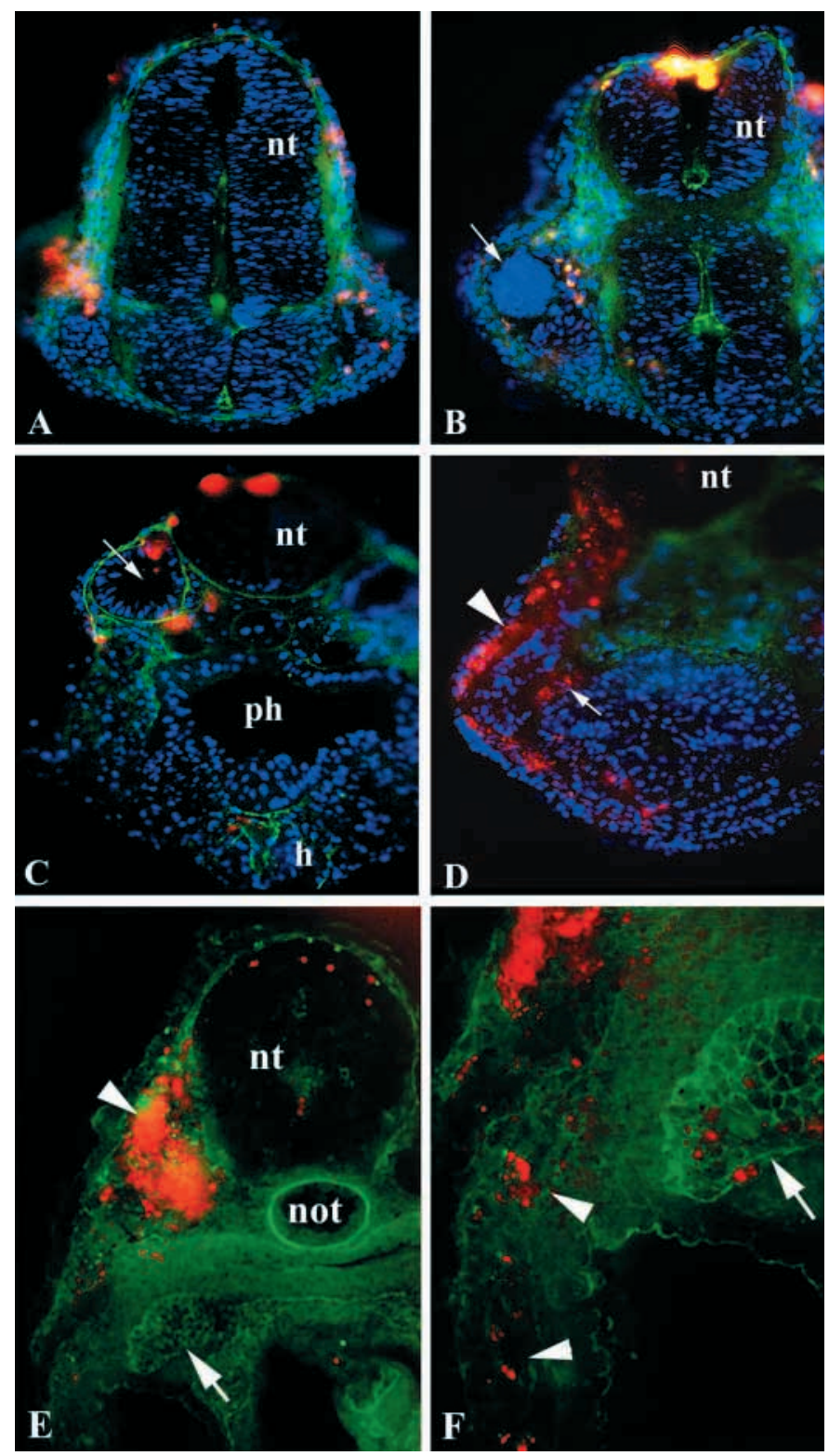

no indication of the precise origin of neural crest cells destined for different branchial arches with respect to the 7 cranial zones (Fig. 1). For this reason, we performed a series of focal injections of DiI into three neural fold sites (corresponding approximately to zones 3,4 and 6; Fig. 2E) of early, stage $15 / 16$ neurulae. Embryos were grown for a further 2.5 days (Fig. 2F-H). In these labeling experiments, we tested whether small regions of neural fold give rise to neural crest cells populating single or multiple branchial arches.

Neural crest cells lying within zone 3 became distributed mainly around the eye. In contrast, cells derived from zone 4 migrated into the position of the prospective maxillary/ mandibular arch and those from zone 6 into the hyoid arch. These results indicate that the first and second branchial arches each receives neural crest cells from small, distinct regions of the neural folds. Since these experiments employed focal injections of DiI, fewer epidermal cells were labeled, allowing us to observe the underlying neural crest cells more clearly. We
Fig. 3. Distribution of DiI-labeled crest cells in the head of axolotl embryos following focal injections of DiI into zones 4-7 of an axolotl neurula. (A) At forebrain levels, labeled crest cells (red) are visible between epidermis and neural tube and at the eye vesicle by 2 days after dye injection. (B) At the level of the optic vesicles (arrow), the injection site is visible in the dorsal neural tube (nt); labeled crest cells spread ventrally and distribute in front of and behind the eye vesicle at 2 days after dye injection. (C) Neural crest cells at the level of the otic vesicle (arrow) are visible adjacent to it and also in the prospective heart region (h) at 2 days post-injection. (D) Dorsal to the pharynx at 3 days after dye injection, we find that the migrating neural crest cell stream becomes divided into a subepidermal stream (presumably pigment cells; arrowhead) and an internal stream (presumably contributing to mesenchymal derivatives; arrow). In A$\mathrm{D}$, transverse vibratome sections $(100 \mu \mathrm{m})$ were counterstained with anti-fibronectin antibodies (to identify tissue borders; green) and DAPI (to identify cell nuclei; blue). In E and F, vibratome sections were counterstained with an antibody to 6-chondroitin sulfate proteoglycan to identify cartilaginous condensations. (E) Although the DiI labeling in this embryo was extensive and had labeled the mesoderm (arrowhead), labeled neural crest cells could be observed migrating ventrally into the gill arches. F shows a higher magnification of the ventral region of the same embryo and illustrates that cells migrate into the gills (arrowheads) and are also found within cartilage (arrow). The same cartilaginous condensation is indicated by an arrow in both $\mathrm{E}$ and $\mathrm{F}$. nt, neural tube; ph, pharynx; $\mathrm{h}$, heart; not, notochord.

observed that neural crest cells close to the neural tube do not undergo extensive mixing.

\section{Transverse sections through the head of focally injected embryos}

Neurulae that had received a single or three adjacent injections into zones 4-7 of the head neural fold were fixed after 1, 2 and 3 days of further development. In vibratome sections through the head region, DiI labeled cells were found at various sites between fore- to hindbrain levels (Fig. 3), for example at the eye and ear vesicles, in the prospective pharynx and heart region and in regions of visceral cartilage. One of the most important findings from these experiments was that migrating cranial neural crest streams split into two divisions, one of which migrates into the branchial arches adjacent to the ectoderm (presumably giving rise to pigment cells), the other migrating along the pharyngeal endoderm (Fig. 3D). Since pharyngeal endoderm is known to be important for cartilage formation (Hörstadius and Sellman, 1946; Epperlein and Lehmann, 1975; Graveson and Armstrong, 1987), these deeper neural crest cells most likely form cartilage of the visceral arches.

\section{Migration and differentiation of cranial neural crest cells in whole mounts using the AP-2 transcription factor as a neural crest cell marker}

As an independent confirmation of our DiI fate mapping experiments, we analyzed the migration of neural crest cells using expression of AP-2 as a marker. AP-2 is a transcription factor expressed in neural crest cells in mice, frogs and chicks (Mitchell et al., 1991; Shen et al., 1997).

\section{Cloning and sequence analysis of Ambystoma AP-2}

We cloned an Ambystoma AP-2 fragment with highest homology to mouse AP-2 and human AP-2 alpha, indicating 

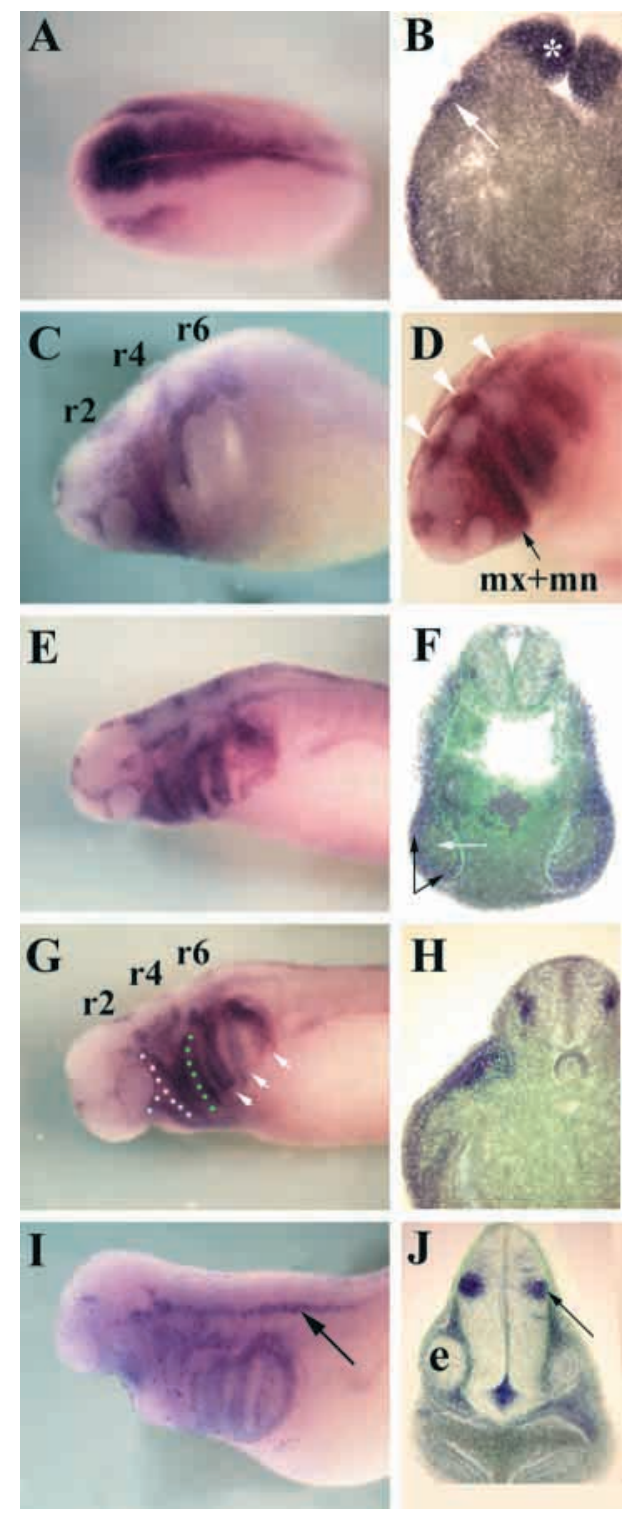

it is the axolotl AP-2 alpha (GenBank accession number AF209775). The published Xenopus AP-2 sequence (Winning et al., 1991) also has high homology with these genes.

\section{In situ hybridizations with AP-2 riboprobe}

In stage 18 embryos (late neurulae), we find expression of AP2 transcripts in dorsomedial parts of the head and anterior trunk neural folds. Although the neural folds are labeled along the entire rostrocaudal axis, we find higher levels of staining in the cranial versus trunk region (Fig. 4A,B). In addition to the neural fold staining, a second domain of weaker staining is found in a stripe of lateral head ectoderm, possibly coinciding with the region of prospective placodal ectoderm.

At stage 22 (Fig. 4C) and, even more clearly at stage 25 (Fig. $4 \mathrm{D}$ ), staining of the neural folds is no longer uniform. Along the rostrocaudal extent of the hindbrain, broader regions of AP2 expression abut regions of more limited AP-2 expression in an alternating pattern that is reminiscent of the even and odd rhombomeres observed in higher vertebrates. In the transverse plane, AP-2 staining in the dorsal cranial neural tube is
Fig. 4. In situ hybridization illustrating the distribution pattern of the transcription factor, AP-2. (A,B). Whole-mount and vibratome section, respectively, through a stage 18 embryo after AP-2 staining. AP-2 staining is distributed in the dorsal and medial parts of head and anterior trunk neural folds (A, and asterisk in B) and in a lateral area of the prospective head region (A, and arrow in B). (C-J) Whole mounts and sections illustrating AP-2 staining as a function of time. C (stage 22), D (stage 25), E (stage 31), G (stage 33) and I (stage 35) demonstrate that there is a continuous decrease of AP-2 staining in the neural folds. By stage 22 (C), unlabeled areas become visible in front of rhombomere 2 (r2) and between rhombomeres 2 and 4 (r4) and 4 and 6 (r6). Staining from the dorsal neural tube continues into the neural crest stream with $\mathrm{r} 2$ being continuous with the maxillary and mandibular arch streams, $\mathrm{r} 4$ with the hyoid arch stream and r6 with a migratory stream common for three gill arches. Concomitant with the decrease of AP-2 staining in the neural folds and branchial arches is the appearance and gradual increase of label in a group of cells in the lateral wall of the head and anterior trunk neural tube, seen most prominently in I and $\mathbf{J}$ (arrows). Transverse sections F,H and $\mathbf{J}$ are overlays of bright-field images of AP-2 staining (blue) with fluorescent images of fibronectin staining (green). The white arrow in $\mathrm{F}$ points to the core of a gill arch and two black arrows indicate AP2-labeled crest cells surrounding it. mx, maxillary arch; mn, mandibular arch; e, otic vesicle; white dots in G, maxillary and mandibular arch neural crest stream; green dots in G, hyoid arch neural crest stream.

continuous with a maxillary/mandibular stream, a more caudal hyoid stream and (at stage 25 ) a gill-arch stream. On the basis of this staining pattern, we propose that AP-2 may be expressed at higher levels in even (r2, r4, r6) than odd-numbered rhombomeres at this stage. Rhombomere 2 (corresponding to zone 4, Fig. 5) gives rise to first branchial arch neural crest, rhombomere 4 (zone 6, Fig. 5) contributes to the hyoid arch and rhombomere 6 (zone 7, Fig. 5) contributes to the gill arches. Although AP-2 expression appears segmental in the hindbrain, we find continuity in staining in the dorsal midline and on the lateral aspect of the embryo, proximal to the individualized neural crest streams.

Between stages 25 to 33, the overall intensity of AP-2 expression in the branchial arches increases, such that distinct maxillary/mandibular, hyoid and three gill arches can be distinguished in later stages (Fig. 4G). By stage 35 (Fig. 4I), AP-2 appears somewhat down-regulated in all the branchial arches. Concomitant with the diminishing AP-2 staining in the neural crest and branchial arches at these stages is an increase of label in the outer lateral wall of the neural tube in head and anterior trunk regions (Fig. 4E,G,I). Furthermore, transverse sections indicate that neural tube staining may be in the RohonBeard cells (Fig. 4J). Between stages 31 and 35, AP-2 staining in the dorsal rhombencephalon diminishes until no staining can be observed.

Taking the results of our DiI fate mapping experiments and the AP-2 expression analysis together, we propose a fate map, illustrated in Fig. 5.

\section{Heterotopic grafting experiments}

To investigate whether premigratory neural crest cells within the head neural folds are committed to a particular branchial arch cell fate, we have performed a series of heterotopic grafting experiments in which small lengths of cranial neural fold or entire cranial neural folds were reversed anteroposteriorly. In addition, we grafted trunk neural folds 
Fig. 5. Fate map of cranial neural crest cells of an axolotl neurula based on labeling with DiI and AP-2 staining. (A) The head neural fold are divided into seven arbitrary zones which give rise to the neural crest streams (same colors) shown in (B) of a stage 33 embryo. The seven zones in B correspond to the seven zones originally defined by Hörstadius and Sellman in 1946 (see Fig. 1B). mx, maxillary arch; mn, mandibular arch; h, hyoid arch; 1-4, four gill arches.

Fig. 6. Heterotopic grafting experiments of cranial neural folds. (A) A schematic diagram illustrating the procedure for anterior cranial neural fold reversals at stage 15 . Anterior neural folds (zones $14)$ are excised, grafted into the same embryo after rotation by $180^{\circ}$ and labeled with DiI. (B) After anterior cranial neural fold reversal, neither zone 1 nor 4 gave rise to a maxillary/mandibular neural crest stream. (C-F) After posterior neural fold displacement, neural crest cells changed their fate and populated new, instead of original, target tissues. (D) For posterior neural fold reversals, zones 5-7 were excised and implanted in a reversed orientation before injection with DiI and/ or CellTracker Green. (C,F) Cells from zone 5 change their branchial arch fate and migrate into gill arches. Similarly, zone 7 cells are able to contribute to $1^{\text {st }}$ and $2^{\text {nd }}$ branchial arches (E and F). (G) Schematic diagram illustrating the methods for full cranial fold reversals. At stage 15, head neural fold fragments comprising zones 1-7 were excised in one neurula and implanted after reversal into the contralateral side of a host neurula. Zones 1 and 7 came to lie far from their original targets while zones 3-5 became displaced only minor distances.

$(\mathrm{H}, \mathrm{J})$ After such reversals, labeled cells from the center of the graft migrate to
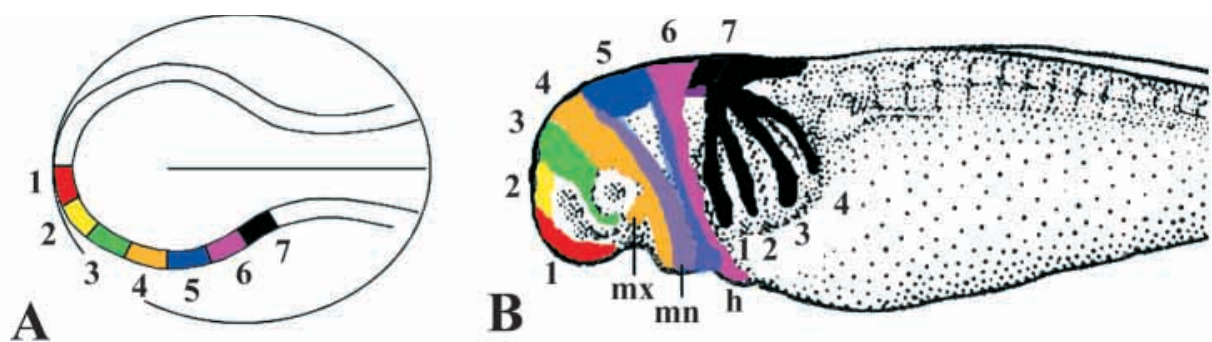

Anterior cranial fold reversals

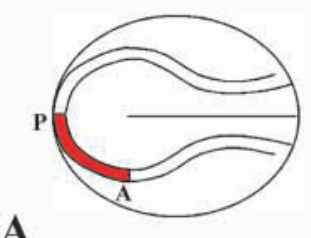

A
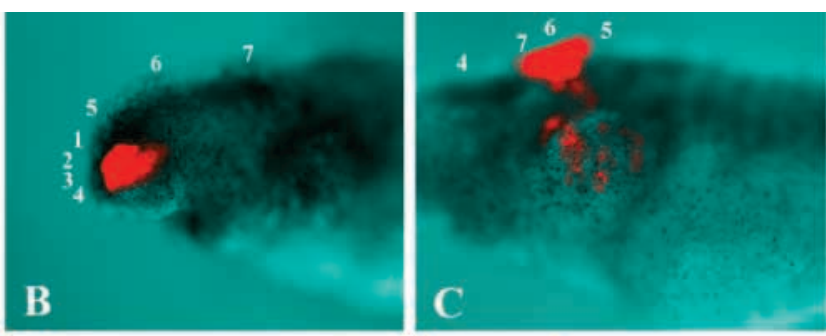

Posterior cranial fold reversals

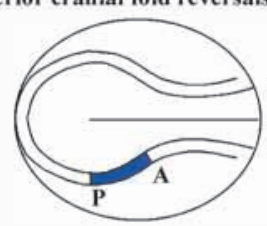

D
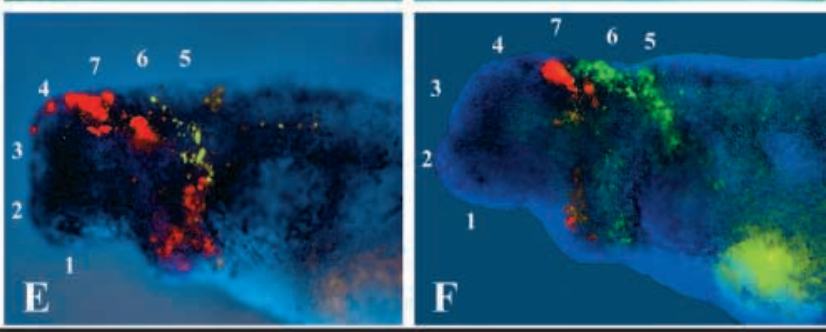

Full-length cranial fold reversals

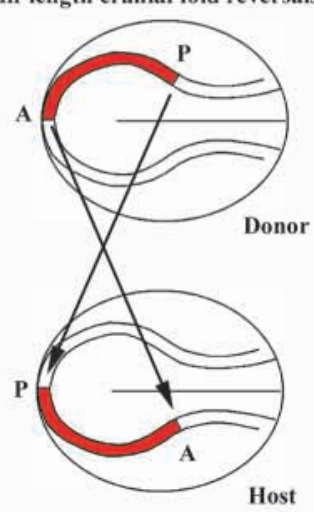

G
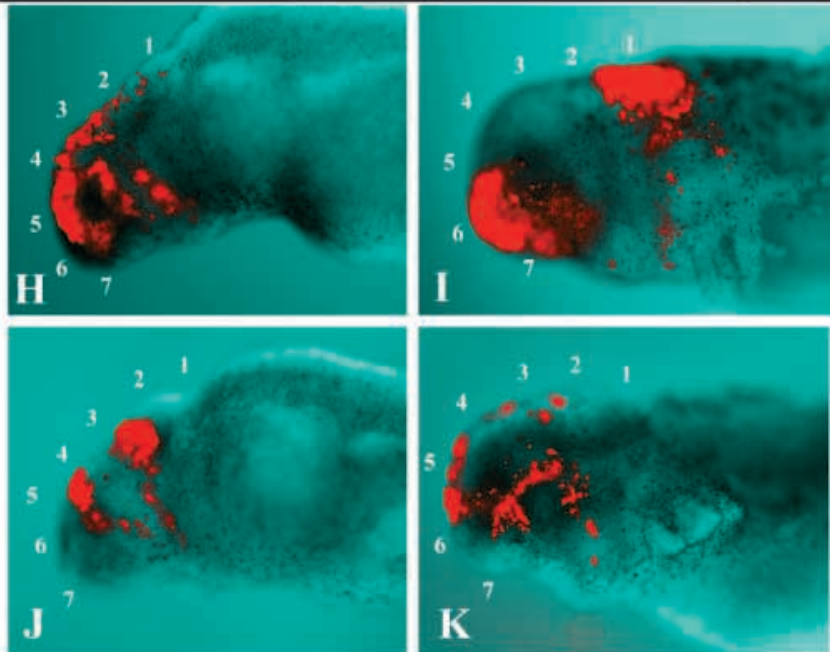

new targets. In contrast, those at the rostral and caudal margins of the graft appeared less able to compensate for their displacement. (I,K) In one case (K) zone 2/3 cells migrated towards the eye and appeared to move rostrally to their original target.

(which normally do not give rise to cartilage) into cranial locations to examine trunk neural crest migratory ability and chondrogenic potential.

\section{Anterior head neural fold reversals}

Zones 1-4 of the head neural fold of an axolotl neurula were excised, rotated $180^{\circ}$ anteroposteriorly and implanted into the same embryo (Fig. 6A) so that zone 1 was transposed to position 4 and vice versa (Fig. 6B). The entire length of neural fold was labeled with DiI prior to rotation. Since zones 1 and 2 are non-skeletogenic and zone 3 gives rise to anterior trabeculae, only zone 4 normally gives rise to neural crest which has extensively migratory ability.

In these operated embryos DiI-labeled neural crest cells from segment 4 , which would normally be fated to form mandibular arch, did not migrate towards the developing mandibular arch as would be appropriate for their level of origin. Thus, the normally migratory segment 4 cells appeared to lose their migratory ability in this new environment. Neural fold cells from segment 1, which normally exhibit little migratory ability (see Figs 1, 5), failed to give rise to cells for the mandibular stream (Fig. 6B). 
Fig. 7. Heterotopic grafting experiments of trunk neural fold into cranial regions. Midtrunk neural fold fragments labeled with DiI were grafted in place of head neural folds (zones 4-5), which normally contribute to the mandibular/hyoid arches. (A-C) As a function of time after grafting, labeled neural crest cells can be observed emigrating from the graft site; they distribute in a random fashion around the head, including a region above the eye vesicle

(arrowheads). Even after 5 days (C) these cells did not align themselves with any of the visceral arch neural crest streams. (D) Transverse sections through head region containing DiI-labeled trunk neural fold graft reveal that some DiI-labeled neural crest cells (red) had migrated in front of and behind the eye vesicle (white arrow), or in areas of developing supportive tissue (bottom of picture). This particular vibratome section was counterstained with DAPI (blue) and immunolabeled with the mesodermal marker 12/101. An absence of 12/101 immunoreactive cells (the green color is background fluorescence) reveals that no trunk mesodermal cells had been inadvertently included in the grafted neural fold fragments, indicating that all DiI-labeled cells were derived from the grafted neural folds. (E) A control section through the trunk of an axolotl
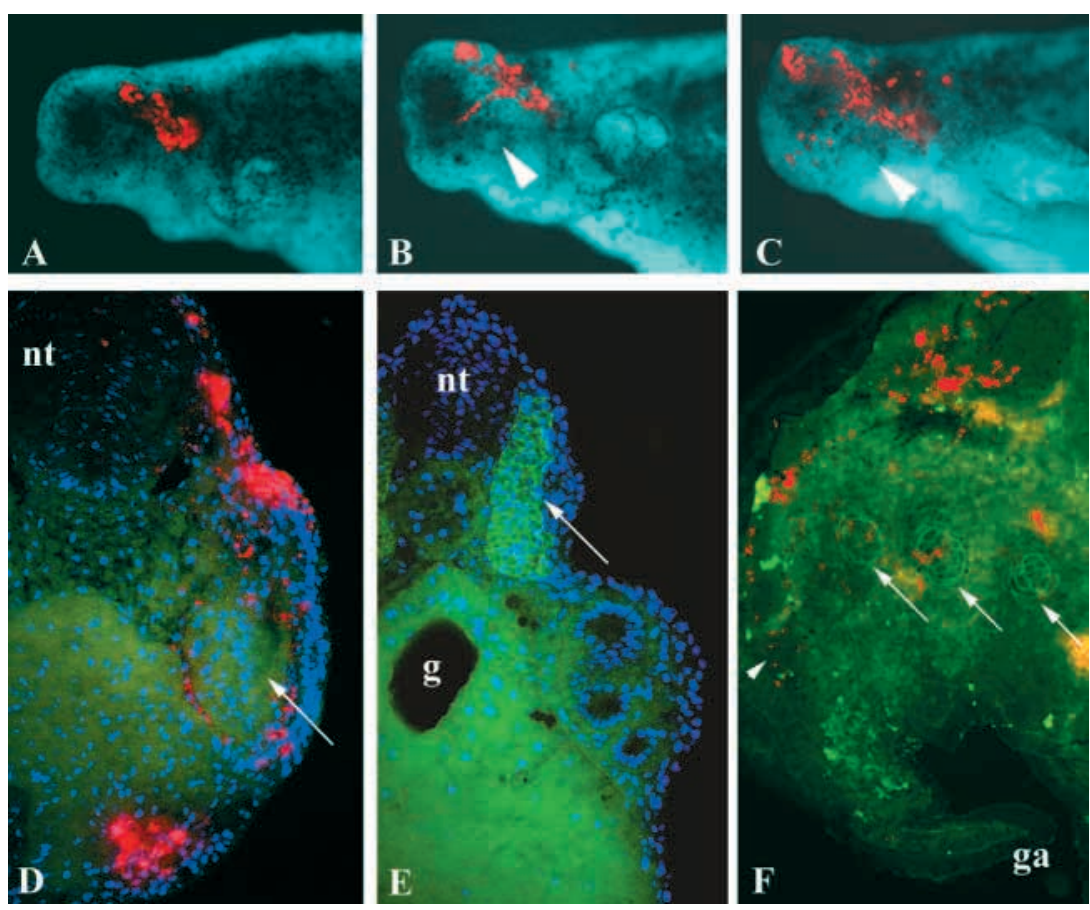
embryo at stage 34 stained with a FITC-conjugated secondary antibody against anti-12/101 reveals specific labeling within the somites (arrow). This section was counterstained with DAPI (blue). (F) While it is clear that trunk neural crest cells would not associate with specific cranial neural crest cell streams, they were found within structures derived from the neural crest, such as cartilaginous elements. In this case, labeled cells (arrowhead) were observed within a gill arch (ga) and also within three cartilaginous nodules (arrows). This section was immunolabeled with an antibody recognizing 6-CSPG to reveal the cartilage. nt, neural tube; ga, gill arch.

\section{Posterior head neural fold reversals}

Head neural fold areas 5-7 of axolotl neurulae were excised and implanted in a reversed orientation (Fig. 6D), as discussed above, so that zone 5 fold lay at position 7 and zone 7 fold cells at position 5 (Fig. 6C,E,F). DiI was used to mark the entire graft in some cases (Fig. 6C), while in others, the borders of the rotated fragment were labeled with DiI and CellTracker Green.

At the time of analysis, we discovered that the graft had shrunk to varying degrees and had come to lie at slightly different rostrocaudal positions. Nevertheless, we found that displaced neural crest cell progenitors could populate tissues appropriate for their new location, and found no evidence of cells migrating towards their original target tissues. For instance, after rotation, cells within zone 5 migrated into the gill arches (Fig. 6C,E,F) which is a tissue that they do not normally encounter. Similarly, zone 7 cells changed their fate and populated the $1^{\text {st }}$ and $2^{\text {nd }}$ branchial arches after neural fold rotations (Fig. 6E,F). As expected, cells from zone 6 migrated to their normal targets as a result of their minimal displacement. In comparing anterior and posterior neural fold reversals, these results suggest that neural crest cells from regions 4-7 compensate after transplantation to a new environment, whereas anterior non-skeletogenic neural folds (regions 1-2) appear unable to acquire migratory ability after displacement.

\section{Entire head neural fold reversals}

Head neural fold fragments comprising regions 1-7 were excised in one neurula and implanted after reversal into the contralateral side of a host neurula (Fig. 6G). As a consequence, cells within zones 1 and 7 come to lie far from their original target site, while neural crest progenitors within zones 3-5 became displaced only minor distances.

Our results indicate that cells at the center of the graft were able to migrate to new targets, consistent with the results above (Fig. 6H,J). In contrast, cells at the rostral and caudal margins of the graft appeared less able to compensate for their displacement and in most cases failed to migrate altogether (Fig. 6I). In one case (Fig. 6K), we found that zone $2 / 3$ cells migrated in close proximity to the eye and, after reaching its dorsal aspect, migrated rostrally towards their original target.

Taken together with the results of small neural fold rotations, our results suggest that premigratory cranial neural crest cells within the neural folds are not committed to a specific branchial arch fate, but can compensate to some extent when displaced short distances from their origin by migrating to a new target tissue. In contrast, when cells are displaced far from their original location, they appear unable to respond appropriately to their new milieu and consequently either fail to migrate or migrate in a disoriented fashion which occasionally brings them to appropriate target sites for their point of origin.

\section{Trunk neural fold fragments}

Head neural fold areas 5-7 of wild-type neurulae (stages 1518) were replaced by midtrunk neural fold fragments in order to study the migratory and differentiative potential of trunk neural crest cells in the head.

In some living embryos, we found that labeled crest cells left the graft caudo-laterally and also became distributed randomly above and behind the eye vesicle (Fig. 7A-C). Even 5 days after 
grafting, these cells did not associate with any of the visceral arch neural crest streams, as would the endogenous neural crest cells. Thus, in the axolotl embryo, trunk crest cells can migrate out in the head region but fail to recognize distinct routes into the visceral arches.

Surprisingly, a few trunk neural crest cells did appear to migrate ventrally towards the branchial arches, as seen clearly in sections through grafted embryos (Fig. 7D,F). At stage 34, labeled neural crest cells were observed in front of and behind the eye vesicle and in areas of developing visceral arches (Fig. 7D). Explantation of trunk neural fold material was carried out without obvious contamination by underlying mesoderm, which can be recognized easily by its white color and larger cells. To rule out the possibility that a few mesodermal cells might occasionally have been included in the graft and subsequently migrated in the head, transverse sections through the head region bearing the graft were stained with a monoclonal antibody against mesodermal cells (12/101; Fig. 7E). As demonstrated in Fig. 7D, the mesodermal marker could neither be observed on DiI-labeled cells nor on unlabeled cells. In some experiments ( $n=4$; data not shown), mesoderm was deliberately included in trunk neural fold grafts, but subsequent analysis revealed that such mesoderm fails to migrate away from the cranial grafts site towards the branchial arches. We conclude that the DiI-labeled cells observed adjacent to the eye and in the gill arches are trunk neural crest cells that have reached these sites by random migration.

Strikingly, trunk neural folds grafted into the head demonstrated the ability to form cartilage after heterotopic grafting. By stage 38, cartilage condensations have formed in host embryos and can be recognized using antibodies to 6chondroitin sulfate proteoglycan (6-CSPG; $n=9)$. DiI-labeled trunk cells were found within the gill branch cartilaginous condensations (Fig. 7F). DiI-labeled cells derived from trunk neural folds became incorporated into these structures and expressed CSPG. Approximately $5-10 \%$ of the cells in these cartilage condensations were DiI labeled.

\section{DISCUSSION}

In this study, we have revisited, using modern cell markers, the issue of the contribution of the neural crest to the visceral skeleton of urodeles, a topic originally examined more than 60 years ago by Hörstadius. Most of the questions asked by Hörstadius and Sellman (1946) examined environmental versus autonomous control of cartilage determination as well as the effects of heterotopic transplantation of the neural folds on head development. Below, we compare the present results to those of Hörstadius, to construct a more accurate map of neural crest fate and potential.

\section{Fate map of the cranial neural crest}

The studies of Hörstadius and Sellman (1946) on the 'determination of the cartilaginous visceral skeleton of urodeles' started in 1936/37 in Triturus and Ambystoma. They were based on earlier findings from Landacre (1921), Stone (1922, 1932), Raven (1931), Ichikawa (1933) and later refined by Chibon (1966) in Pleurodeles, another urodele. To establish a preliminary fate map, Hörstadius and Sellman used vital dyes, as devised by Vogt (1925), to follow the normal patterns of cranial neural crest migration (see Fig. 1). Agar pieces stained with Nile Blue sulfate and Neutral Red were placed directly onto the neural folds of neurulae in order to label presumptive neural crest. The chief findings from these studies are that neural crest cells migrate from the neural folds as distinct streams en route to the branchial arches.

In the present study, focal injections of DiI into the neural folds confirmed these earlier findings by demonstrating a maxillary/mandibular stream, a hyoid stream and 2-3 streams for the gill arches. Furthermore, using focal injections, we show that neural crest cells do not undergo extensive mixing on their initial route of migration (Fig. 2E-H). Furthermore, we show that neural crest cells associate with epithelium as they migrate into the branchial arches. We propose that those neural crest cells associated with the superficial ectoderm develop into pigment cells, while those in contact with the pharyngeal endoderm differentiate into cartilage.

By cloning axolotl AP-2, we have established an excellent molecular marker for the cranial neural crest. In situ hybridization with AP-2 shows the pattern of the premigratory neural crest cells and their early routes of migration more clearly than can be observed with DiI labeling. AP-2 is expressed in the head crest in a number of genera, including amphibian, chick and mouse (Mitchell et al., 1991; Shen et al., 1997). This transcription factor is important for the regulation of facial structures and also for other genes expressed by neural crest cells including Hoxa2 (Maconochie et al., 1999).

In the axolotl, AP-2 is expressed within the premigratory and migrating neural crest cells as well as in postmigratory cells within the branchial arches. In the hindbrain, AP-2 expression in the neural tube is segmental, appearing more prominently in even numbered rhombomeres ( $\mathrm{r} 2, \mathrm{r} 4, \mathrm{r} 6)$. There is apparent continuity in the cell streams emanating from the even numbered rhombomeres and giving rise to individual branchial arch crest streams. Interestingly, scanning electron microscope analysis of axolotl embryos has shown that neural crest cells exist as a continuous flattened epithelium along the length of the rhombencephalic neural tube at these stages (Epperlein and Löfberg, 1993), suggesting that AP-2 is not expressed in all premigratory neural crest populations.

The combination of the AP-2 staining pattern and DiI labeling would suggest the following fate map of neural crest cells (Fig. 5).

(i) First branchial arch neural crest arises from a distinct region of the neural folds, designated zone 4 (after Hörstadius and Sellman, 1946). These neural crest cells express AP-2 as they migrate ventrally, where they split into maxillary and mandibular streams.

(ii) Second branchial arch neural crest arises from about the anterior three quarters of zone 6 and migrates as a distinct AP2-positive stream towards the hyoid arch.

(iii) Third to sixth branchial arch neural crest cells arise as a single stream from about the posterior three quarters of zone 7 before separating into streams corresponding to each of the gill arches.

(iv) Neural crest cells do not seem to migrate laterally from either zone 5 or a region lying between zones 6 and 7 . Interestingly, both regions express AP-2 at very low levels, raising the possibility that these two areas do not contribute to the branchial arches. 


\section{Regulatory ability of head neural crest segments}

We carried out heterotopic grafting experiments with different head neural fold fragments and labeled them with DiI or, in some cases, both with DiI and CellTracker Green, in order to examine the degree of plasticity versus prepattern in cranial neural crest cells. We found that cranial neural crest cells can compensate for small displacements by migrating appropriately for their new environment (Fig. 6). However, when cells are displaced far from their premigratory location, they do not migrate normally for their new environment, appearing to undergo random movements and only rarely finding their original destinations. These data indicate that cranial neural crest cells have some intrinsic properties regarding their rostrocaudal position, but also have some degree of plasticity in adapting to new surroundings. Our results suggest that the anterior-most neural folds (regions 13) do not contribute to the neural crest even after heterotopic transplantation.

Our neural fold transpositions are supported by recent results in other systems. For example, rhombomere rotations in the chick show that the neural crest can partially compensate for small changes by migrating to branchial arches appropriate for their new location (Sechrist et al., 1994; Saldivar et al., 1997). Similarly, Hunt et al. (1998) show that changing the spatial organization of the entire chick rhombencephalic neural tube leads to neural crest cells migrating towards branchial arches closest to their new location. This type of operation is analogous to the posterior cranial neural fold reversals performed in the present study.

In general, the results of tissue grafts and ablations have demonstrated that some aspects of neural crest cell migration and differentiation are plastic, whereas others are somewhat fixed. Neural crest adjacent or contralateral to the operation site, or the neural tube itself might be involved in regulatory events. For example, removal of the neural crest by the ablation of a small portion of the dorsal neural tube at midbrain and trunk levels results in a normal embryo (Yntema and Hammond, 1945, 1947; McKee and Ferguson, 1984). This has been taken as evidence that neighboring neural crest cells, both rostral and caudal to the ablated region, repopulate the extirpated segments. Ablation of midbrain neural folds in chick embryos leads to normal development of neural crest-derived structures (McKee and Ferguson, 1984). Interestingly, the neural crest cells that 'fill-in' from ectopic regions appear to readjust their Hox code after such an operation (Hunt et al., 1995). In addition to compensation by neighboring populations, the neural tube itself can regulate to reform neural crest after such ablations, but only for a limited period of time in development (Scherson et al., 1993; Sechrist et al., 1995).

\section{The potential of the trunk crest in the head}

We and other authors (Raven, 1931; Hörstadius and Sellman, 1946) grafted trunk neural crest cells into the head region of axolotl neurulae in order to investigate their migratory and differentiative potential in a new environment. Whereas previous studies observed no indications of migration and cartilage formation, we found evidence for both events. DiIlabeled trunk crest cells migrated out in a random fashion and became distributed above and behind the eye vesicle. They did not, however, associate with any of the neural crest streams as could be demonstrated in whole mounts (Fig. 7A-C). In transverse sections through the head region bearing the graft (Fig. 7D,F), DiI-labeled cells were found in various places under the epidermis. Importantly, they also were observed within cartilaginous elements of the head skeleton (Fig. 7F). Thus, most of the grafted trunk neural crest probably gives rise to pigment cells (subepidermal location) and neurons (not identified) in addition to skeletogenic derivatives. One possible reason why previous studies failed to find incorporation of trunk neural crest into cartilage is that neural crest cells were labeled with vital dyes that rapidly dilute to sub-detectable levels in dividing cells. If chondrogenic neural crest cells reach their targets by migrating deep within the branchial arches, it would be difficult to observe them in whole mounted embryos, or even in relatively thick transverse sections.

Various differences exist between trunk and head neural crest such as their cellular shape (elongated versus polygonal), migratory pathways (between somites and epidermis or somites and neural tube/notochord in the trunk and between epidermis and endoderm in the head), mode of migration (single cells versus cellular tongues) and the derivatives generated. The production of cartilage has classically been thought to be a property of the cranial neural crest only, since trunk neural crest did not produce cartilage when transplanted into the cranial region (Raven, 1931; Hörstadius and Sellman, 1946; Noden, 1975; Nakamura and Ayer-Le Lièvre, 1982; Graveson et al., 1995). For instance, in the axolotl, previous studies have failed to show that trunk neural crest cells form cartilage when transplanted to the head (Raven, 1931; Graveson et al., 1995). However, trunk neural crest cells in amphibia do appear to contribute to the mesenchyme of the dorsal fin (DuShane, 1935; Collazo et al., 1993), though most mesenchyme is mesodermally derived. The formation of head cartilage depends upon various environmental factors, specifically on an interaction with endodermal and ectodermal epithelia (Tyler and Hall, 1977; Bee and Thorogood, 1980; Hall, 1981, 1987; Hall and Hörstadius, 1988).

The results of our heterotopic grafting experiments suggest that trunk neural crest cells do not migrate along normal cranial neural crest pathways, but move in a disoriented fashion. As a consequence, the cells appear to distribute randomly. Despite this random migration, they appear to encounter specific cues that allow them to form cartilage and contribute to elements of the visceral skeleton. Our results provide the first evidence that cartilage can develop from trunk neural crest cells in vivo. Thus, it seems that the migratory ability of trunk neural crest cells is uncoupled from their developmental potential.

This work was supported by a Howard Hughes Medical Institute Research Resources Grant and a James H. Zumberge Research and Innovation Fund (M. A. J. S.), by NS36585 and NS34671 to M. B. F. and the Deutsche Forschungsgemeinschaft (Ep8/7-1) to H.-H. E. The 12/101 monoclonal antibody, developed by Jeremy Brockes, was obtained from the Developmental Studies Hybridoma Bank maintained by The University of Iowa, Department of Biological Sciences, Iowa City, IA 52242, USA.

\section{REFERENCES}

Baroffio, A., Dupin, E., and LeDouarin, N. M. (1991). Common precursors for neural and mesectodermal derivatives in the cephalic neural crest. Development 112, 301-305. 
Bee, J. and Thorogood, P. (1980). The role of tissue interactions in the skeletogenic differentiation of avian neural crest cells. Dev. Biol. 78, 4762.

Bordzilovskaya, N. P., Dettlaff, T. A., Duhon, S. T., and Malacinski, G. M. (1989). Developmental-stage series of axolotl embryos. In The Developmental Biology of the Axolotl (ed. J. B. Armstrong and G. M. Malacinski.), pp. 201-219. New York: Oxford University Press.

Bronner-Fraser, M. and Fraser, S. E. (1988). Cell lineage analysis reveals multipotency of some avian neural crest cells. Nature 335, 161-164.

Bronner-Fraser, M. and Fraser, S. (1989). Developmental potential of avian trunk neural crest cells in situ. Neuron 3, 755-766.

Chibon, P. (1966). Analyse expérimentale de la régionalisation et des capacités morphogénètiques de la crête neurale chez l' amphibien urodéle Pleurodeles waltlii. Michah. Mem. Soc. Zool. Fr. 36, 1-107.

Collazo, A., Bronner-Fraser, M. and Fraser, S. E. (1993). Vital dye labeling of Xenopus laevis neural crest cells reveals multipotentiality and novel pathways of migration. Development 118, 363-376.

DuShane, G. P. (1935). An experimental study of the origin of pigment cells in Amphibia. J. Exp. Zool. 19, 1-31.

Epperlein, H. H. and Lehmann, R. (1975). The ectomesenchymalendodermal interaction system (EEIS) of Triturus alpestris in tissue culture. 2. Observations on the differentiation of visceral cartilage. Differentiation 4, 159-174.

Epperlein, H. H. and Löfberg, J. (1990). The development of the larval pigment patterns in Triturus alpestris and Ambystoma mexicanum. Adv. Anat. Embryol. Cell Biol. 118, 1-101.

Epperlein, H. H. and Löfberg, J. (1993). The development of the neural crest in amphibians. Ann. Anat. 175, 483-499.

Graveson, A. C. and Armstrong, J. B. (1987). Differentiation of cartilage from cranial neural crest in the axolotl (Ambystoma mexicanum). Differentiation 35, 16-20.

Graveson, A. C., Hall, B. K. and Armstrong, J. B. (1995). The relationship between migration and chondrogenic potential of trunk neural crest cells in Ambystoma mexicanum. Wilhelm Roux's Arch. Dev. Biol. 204, 477-254.

Hall, B. K. (1981). The induction of neural crest-derived cartilage and bone by embryonic epithelia: an analysis of the mode of action of an epithelialmesenchymal interaction. J. Embryol. Exp. Morphol. 64, 305-320.

Hall, B. K. (1987). Development of the mandibular skeleton in the embryonic chick as evaluated using the DNA-inhibiting agent 5-fluoro-2'-deoxyuridine. J. Craniofac. Genet. Dev. Biol. 7, 145-159.

Hall, B. K. (1999). The Neural Crest in Development and Evolution. New York: Springer-Verlag New York Inc.

Hall, B. K. and Hörstsadius, S. (1988). The Neural Crest. London: Oxford University Press.

Henrique, D., Adam, J., Myat, A., Chitnis, A., Lewis, J. and Ish-Horowicz, D. (1995). Expression of a Delta homologue in prospective neurons in the chick. Nature 375, 787-790.

Honig, M. G. and Hume, R. I. (1989). Carbocyanine dyes. Novel markers for labelling neurons. Trends Neurosci. 12, 336-338.

Hörstadius, S. and Sellman, S. (1946). Experimentelle Untersuchungen über die Determination des knorpeligen Kopfskelettes bei Urodelen. Nov. Act. Reg. Soc. Scient. Ups. Ser. IV 13, 1-170.

Hunt, P., Ferretti, P., Krumlauf, K., and Thorogood, P. (1995). Restoration of normal Hox code and branchial arch morphogenesis after extensive deletion of hindbrain neural crest. Dev. Biol. 168, 584-597

Hunt, P., Clarke, J. D. W., Buxton, P., Ferretti, P. \& Thorogood, P. (1998). Stability and plasticity of neural crest patterning and branchial arch Hox code after extensive cephalic crest rotation. Dev. Biol. 198, 82-104.

Ichikawa, M (1933). Experiments on the gill formation in the urodelan Triturus. Mem. Coll. Sci. Kyoto Imp.Univ. Ser. B, 9, 47.

Landacre, F. L. (1921). The fate of the neural crest in the head of the urodeles. J. Comp. Neurol. 33, 1-43.

Le Douarin, N. M. (1982). The Neural Crest. Cambridge: Cambridge University Press.

Maconochie, M., Krishnamurthy, R., Nonchev, S., Meier, P., Manzanares, M., Mitchell, P. J. and Krumlauf, R. (1999). Regulation of Hoxa2 in cranial neural crest involves members of the $A P-2$ family. Development 126, 1483-1494.

McKee, G. J. and Ferguson, M. W. (1984). The effects of mesencephalic neural crest cell extirpation on the development of chicken embryos. J. Anat. 139, 491-512.

Mitchell, P. J., Timmons, P. M., Hebert, J. M., Rigby, P. W. Tjian, R.
(1991). Transcription factor AP-2 is expressed in neural crest cell lineages during mouse embryogenesis. Genes Dev. 5, 105-119.

Nakamura, H. and Ayer-Le Lièvre, C. (1982). Mesectodermal capabilities of the trunk neural crest of birds. J. Embryol. exp. Morphol. 70, 1-18.

Noden, D. M. (1975). An analysis of the migratory behavior of avian cephalic neural crest cells. Dev. Biol. 42, 106-130.

Raven, C. P. (1931). Zur Entwicklung der Ganglienleiste. I. Die Kinematik der Ganglienleistenentwicklung bei den Urodelen. Wilhelm Roux Arch. EntwMech.Org. 125, 210-293.

Reissmann, E., Ernsberger, U., Francis-West, P. H., Rueger, D., Brickell, P. M. and Rohrer, H. (1996). Involvement of bone morphogenetic protein4 and bone morphogenetic protein-7 in the differentiation of the adrenergic phenotype in developing sympathetic neurons. Development 122, 2079 2088.

Saldivar, J. R., Sechrist, J. W., Krull, C. E., Ruffins, S. and BronnerFraser, M. (1997). Dorsal hindbrain ablation results in rerouting of neura crest migration and changes in gene expression, but normal hyoid development. Development 124, 2729-2739.

Scherson, T., Serbedzija, G. N., Fraser, S. and Bronner-Fraser, M. (1993) Regulative capacity of the cranial neural tube to form neural crest Development 118, 1049-1062.

Sechrist, J. Scherson, T. and Bronner-Fraser, M. (1994). Rhombomere rotation reveals that multiple mechanisms contribute to the segmental pattern of hindbrain neural crest migration. Development 120, 1777-1790.

Sechrist, J., Nieto, M. A., Zamanian, R. T. and Bronner-Fraser, M. (1995) Regulative response of the cranial neural tube after neural fold ablation: spatiotemporal nature of neural crest regeneration and up-regulation of Slug. Development 121, 4103-4115.

Selleck, M. A. J. and Bronner-Fraser, M. (1995). Origins of the avian neural crest: the role of neural plate-epidermal interactions. Development 121, 525538

Serbedzija, G. N., Bronner-Fraser, M. and Fraser, S. E. (1989). A vital dye analysis of the timing and pathways of avian trunk neural crest cell migration. Development 106, 809-816.

Serbedzija, G., Bronner-Fraser, M. and Fraser, S. E. (1992). Vital dye analysis of cranial neural crest cell migration in the mouse embryo. Development 116, 297-307.

Shah, N. M., Marchionni, M. A., Isaacs, I., Stroobant, P. and Anderson, D. J. (1994). Glial growth factor restricts mammalian neural crest stem cells to a glial fate. Cell. 77, 349-60.

Shah, N. M., Groves, A. K. and Anderson, D. J. (1996). Alternative neural crest cell fates are instructively promoted by TGFbeta superfamily members. Cell 85, 331-343.

Shen, H., Wilke, T., Ashique, A. M., Narvey, M., Zerucha, T., Savino, E., Williams, T. and Richman, J. M. (1997). Chicken transcription factor AP2: cloning, expression and its role in outgrowth of facial prominences and limb buds. Dev. Biol. 188, 248-266.

Steinberg, M. S. (1957). A nonnutrient culture medium for amphibian embryonic tissue. Carnegie Inst. Wash. Year b. 56, 347-348.

Stone, L. S. (1922). Experiments on the development of the cranial ganglia and the lateral line sense organs in Amblystoma punctatum. J. Exp. Zool. 35, 421-496.

Stone, L. S. (1932). Transplantation of hyobranchial mesentoderm, including the right lateral anlage of the second basibranchium in Amblystoma punctatum. J. Exp. Zool. 62, 109-123.

Tyler, M. S. and Hall, B. K. (1977). Epithelial influences on skeletogenesis in the mandible of the embryonic chick. Anat. Rec. 188, 229-239.

Varley, J. E., Wehby, R. G., Rueger, D. C. and Maxwell, G. D. (1995). Number of adrenergic and islet-1 immunoreactive cells is increased in avian trunk neural crest cultures in the presence of human recombinant osteogenic protein-1. Dev. Dyn. 203, 434-447.

Vogt, W. (1925). Gestaltungsanalyse am Amphibienkeim mit örtlicher Vitalfärbung. I. Methodik und Wirkungsweise der örtlichen Vitalfärbung mi Agar als Farbträger. Wilhelm Roux Arch. EntwMech.Org. 106, 542-610.

Winning, R. S., Shea, L. J., Marcus, S. J. and Sargent, T. D. (1991). Developmental regulation of transcription factor AP-2 during Xenopus laevis embryogenesis. Nucl. Acids Res. 19, 3709-3714.

Yntema, C. L. and Hammond, W. S. (1945). Depletions and abnormalities in the cervical sympathetic system of the chick following extirpation of the neural crest. J. Exp. Zool. 100, 237-263.

Yntema, C. L. and Hammond, W. S. (1947). The development of the autonomic nervous system. Biol. Rev. 22, 344-357. 